J. Clin. Chem. Clin. Biochem.

Vol. 16, 1978, pp. 613-619

\title{
Adaptation sur GSA II Greiner du dosage des triglycérides par voie entièrement enzymatique Application à l'étude de leur conservation et de certaines interférences analytiques
}

\author{
Par Josiane Steinmetz \\ Laboratoire du Centre de Médecine Préventive (Directeur: Prof. G. Siest), \\ 2 avenue du Doyen Jacques Parisot, 54500 Vandoeuvre-les-Nancy, France, et
}

\section{Edwige Panek}

Laboratoire de Biochimie Pharmacologique, Faculté des Sciences Pharmaceutiques et Biologiques, 7 rue Albert Lebrun, 54000 Nancy, France

(Reçu le 11 avril/4 juillet 1978)

Résumé: L'adaptation du dosage des triglycérides par voie entièrement enzymatique a èté réalisée sur GSA II Greiner. La méthode ne nécessite que $20 \mu$ de prise d'essai de matériel biologique. Le temps de la réaction est court: 8 min à $37^{\circ} \mathrm{C}$. Le coefficient de variation de la méthode ne dépasse pas $6 \%$ sur une période de un mois. La linéarité des mesures est vérifiée jusqu'à un taux de $4 \mathrm{mmol} / \mathrm{l}$. Il a été montré que le taux de glycérol libre doit être mesuré systématiquement et déduit du glycérol total pour assurer l'exactitude des résultats. La stabilité des triglycérides plasmatiques a été vérifiée en fonction de la durée et de la température de conservation $\left(+4{ }^{\circ} \mathrm{C} \mathrm{et}-196{ }^{\circ} \mathrm{C}\right)$. Le glycérol libre augmente en fonction du temps, alors que le taux de triglycérides demeure constant. Nous n'avons constaté aucune interférence analytique en présence d'hémoglobine ou de médicaments, en particulier hypolipémiants et hypoglycémiants.

Adaptation of an entirely enzymatic triglyceride assay to the GSA II Greiner. Application to the study of the stability of triglycerides, and factors causing interference in the analysis

Summary: The assay of triglycerides by an entirely enzymatic pathway was adapted for use on the GSA II Greiner. The method requires only $20 \mu \mathrm{l}$ of biological material. The reaction time is short: $8 \mathrm{~min}$ at $37^{\circ} \mathrm{C}$. The coefficient of variation does not exceed $6 \%$ over a period of one month. Linearity of the measurements was verified up to a level of $4 \mathrm{mmol} / \mathrm{l}$. It was shown that the free glycerol level should be systematically measured and deducted from that of the total glycerol to assure accurate results. The stability of the plasma triglycerides was investigated as a function of the length and temperature of storage $\left(+4^{\circ} \mathrm{C}\right.$ and $\left.-196^{\circ} \mathrm{C}\right)$. The free glycerol increases as a function of time, whereas the level of triglycerides stays constant. Haemoglobin and various medicaments, in particular, hypolipemiants and hypoglycemiants, did not interfere.

\section{Introduction}

L'introduction des méthodes entièrement enzymatiques du dosage des triglycérides a été un progrès considérable dans le domaine de la chimie clinique, du fait de leur simplicité et de leur rapidité (1). Leur adaptạtion sur des appareils automatiques en flux continu $(2,3,4)$ ou semiautomatiques (5), tels que les analyseurs centrifuges (6-11) a été proposée. Nous nous sommes intéressés à l'automatisation de ce principe de dosage sur un autre appareil automātique de type discret, le GSA II Greiner, en orientant nos essais vers la recherche de la précision et de l'exactitude des résultats. Ceux-ci ont donc été comparés à une méthode chimique, la méthode de Soloni (12), considérée par de nombreux auteurs, dont le "Lipid-Research Clinic Laboratory, Center for Disease Control, Atlanta), comme la méthode sélectionnée du dosage dés triglycérides. Connaissant les performances analytiques de l'appareil, la stabilité des triglycérides en fonction du temps de conservation à $-196^{\circ} \mathrm{C}$ et $4^{\circ} \mathrm{C}$ a été étudiée. Enfin, noùs avons vérifié l'interférence analy tique de quelques médicaments, de l'héparinate de lithium êt de l'hémoglobine. 


\section{Matériel et méthodes}

Dosage des trigly cerides

\section{Méthode enzymatique}

Les triglycérides sont hydrolysés par l'association d'une lipase et d'une estérase (1). Le glycérol libéré est dosé selon la réaction de Kreutz (13).

Glycérol + ATP $\stackrel{\text { Glycérokinase }}{\longrightarrow}$ sn-glycérol-3-P + ADP

$\mathrm{ADP}+$ phosphoenolpyruvate $\stackrel{\text { Pyruvate kinase }}{\longrightarrow}$ ATP + pyruvate

Pyruvate $+\mathrm{NADH}+\mathrm{H}^{+} \stackrel{\text { Lactate dehydrogénase }}{\longrightarrow}$ lactate $+\mathrm{NAD}^{+}$

\section{Réactifs}

Nous avons utilisé les coffrets prêts à l'emploi Boehringer Mannheim (Réf. 15725). Les concentrations des réactifs dans le mélange réactionnel et dans le volume final sont les suivantes:

Réactif R1: Mélange réactionnel:

\begin{tabular}{|c|c|c|}
\hline & $\begin{array}{l}\text { Concentrations } \\
\text { initiales } \\
(\mathrm{mmol} / \mathrm{l})\end{array}$ & $\begin{array}{l}\text { Concentrations } \\
\text { finales dans le } \\
\text { mélange } \\
\text { réactionnel } \\
(\mathrm{mmol} / \mathrm{l})\end{array}$ \\
\hline $\begin{array}{l}\text { Tampon phosphate } \\
\text { de potassium } \mathrm{pH} 7,0\end{array}$ & 47,5 & 32,5 \\
\hline Sulfate de magnésium & 3,8 & 2,6 \\
\hline $\begin{array}{l}\text { Dodécyl sulfate } \\
\text { de sodium }\end{array}$ & 0,33 & 0,23 \\
\hline $\mathrm{NADH}$ & 0,25 & 0,17 \\
\hline ATP & 0,55 & 0,38 \\
\hline $\begin{array}{l}\text { Phosphoenol- } \\
\text { pyruvate }\end{array}$ & 0,45 & 0,31 \\
\hline $\begin{array}{l}\text { Lactate } \\
\text { dehydrogénase }\end{array}$ & $\geqslant 7500 \mathrm{U} / 1$ & $\geqslant 5136 \mathrm{U} / 1$ \\
\hline Pyruvate kinase & $\geqslant 1250 \mathrm{U} / 1$ & $856 \mathrm{U} / 1$ \\
\hline Lipase & $\geqslant 100000 \mathrm{U} / 1$ & $\geqslant 68000 \mathrm{U} / 1$ \\
\hline Estérase & $750 \mathrm{U} / 1$ & $\geqslant 514 \mathrm{U} / 1$ \\
\hline
\end{tabular}

Stabilité: 2 jours à $+4{ }^{\circ} \mathrm{C}$

Réactif R2: Suspension de glycérokinase $\geqslant 4500 \mathrm{U} / 1$ Concentration dans le volume final $\geqslant 678 \mathrm{U} / 1$ Stabilité: 4 jours à $+4^{\circ} \mathrm{C}$

\section{Mode opératoire}

$20 \mu \mathrm{l}$ de plasma sont incubés à $37^{\circ} \mathrm{C}$ pendant $8 \mathrm{~min}$ avec $500 \mu \mathrm{l}$ de mélange réactionnel (R1) et $110 \mu \mathrm{l}$ de gly cérokinase (R2). En parallèle, un blanc réactif est mesuré, omission faite de l'addition de glycérokinase (tube 2 ). Le protocole de dosage est décrit dans le tableau 1, suivant la présentation proposée par le fabricant.

Tab. 1. Schéma de dosage des triglycérides par méthode enzymatique sur GSA II Greiner.

\begin{tabular}{|c|c|c|c|c|c|}
\hline & \multirow{2}{*}{ REACTIFS } & \multirow{2}{*}{$\begin{array}{l}\text { TEMPS } \\
\min \times 10\end{array}$} & \multicolumn{2}{|c|}{ VOLUMES [ $\mu$ l] } & \multirow{2}{*}{$\begin{array}{l}\text { ESSAIS } \\
1 \text { 而 }\end{array}$} \\
\hline & & & 1 & ॥I & \\
\hline & $5+D F(x+100)$ & 100 & 120 & 120 & \\
\hline R1 & Mélange réactionnel & 80 & 500 & 500 & \\
\hline R2 & Glycérokinase & 61 & 110 & - & \\
\hline \multirow[t]{3}{*}{ R3 } & Eau distillée & 21 & - & 110 & \\
\hline & . & & & & \\
\hline & LECIURE & 0 & 730 & 730 & $\lambda=365 \mathrm{~nm}$ \\
\hline
\end{tabular}

La densité optique initiale (sans addition de glycérokinase) est lue dans le tube 2 et la densité optique finale dans le tube 1 .

\section{Calculs}

La concentration en triglycérides est calculée d'après la formule:

$$
\begin{aligned}
& c(\mathrm{mmol} / \mathrm{l})=\Delta \mathrm{A} \times \frac{1}{\epsilon} \times \frac{\mathrm{VF}}{\mathrm{VI}} \\
& \epsilon(\mathrm{NADH}, 365 \mathrm{~nm})=3,4 \times 10^{6} \mathrm{~cm}^{2} \mathrm{~mol}^{-1} \\
& \mathrm{VF}=730 \mu \mathrm{l} \\
& \mathrm{VI}=20 \mu \mathrm{l} \\
& \mathrm{c} \equiv \Delta \mathrm{A} \times 10,73
\end{aligned}
$$

L'appareil est étalonné avec des solutions étalon de gly cérol en solution aqueuse. Le coefficient multiplicatif $(10,73)$ est affiché. Le résultat final est calculé automatiquement par l'appareil et transmis sous forme de carte perforée.

\section{Mẹthode chimique}

Nous avons adopté la méthode de Soloni (12) en technique manuelle. Cette méthode est basée sur l'extraction sélective des triglycérides paŕ le mélange nonane-isopropanol. Après transestérification par l'ethoxide de sodium, le glycérol est transformé par oxydation périodique en formaldehyde qui est dosé sũivant là réactiọn dè Hantżsch.

La trioléine utilisée pour l'étalonnage de la méthode est un produit Sigma (T7502).

Dosage du glycerol libre

Le glycérol libre est dosé par la méthode enzymatique de Kreutz (13) sur GSA II Greiner.

\section{Réactifs}

\begin{tabular}{|c|c|c|}
\hline & $\begin{array}{l}\text { Concentrations } \\
\text { initiales } \\
(\mathrm{mmol} / \mathrm{l})\end{array}$ & $\begin{array}{l}\text { Concentrations } \\
\text { finales dans le } \\
\text { mélange } \\
\text { réactionnel } \\
(\mathrm{mmol} / \mathrm{l})\end{array}$ \\
\hline $\begin{array}{l}\text { Tampon } \\
\text { triéthanolamine } \\
\text { pH } 7,6\end{array}$ & 95,2 & 67 \\
\hline $\begin{array}{l}\text { Sulfate de } \\
\text { magnésium }\end{array}$ & 3,8 & 2,6 \\
\hline NADH & 0,24 & 0,17 \\
\hline ATP & 1,32 & 0,93 \\
\hline $\begin{array}{l}\text { Phosphoenol- } \\
\text { pyruvate }\end{array}$ & 0,44 & 0,31 \\
\hline $\begin{array}{l}\text { Lactate } \\
\text { dehydrogénase }\end{array}$ & $\geqslant 6400 \mathrm{U} / \mathrm{l}$ & $\geqslant 4500 \cdot \mathrm{U} / \mathrm{l}$ \\
\hline Pyruvate kinase & $\geqslant 1.040 \mathrm{U} / 1$ & $>730 \mathrm{U} / \mathrm{l}$ \\
\hline
\end{tabular}

Nous avons utilisé les coffrets Boehringer Manniheim (Réf. 124966) préconisés pour le dosage du glycérol libéré par hydrolyse alcaline.

Réactif R1: Mélange réactionnel:

Réactif R2: Suspension de glycérokinase $>3000 \mathrm{U} / 1$ Concentration dans le volume final $>210 \mathrm{U} / 1$ Stabilité: 4 jours à $+4^{\circ} \mathrm{C}$. 


\section{Mode opératoire}

Le protocole de dosage est décrit dans le tableau 2:

Tab. 2. Schéma de dosage du glycérol libre par méthode enzymatique sur GSA II Greiner.

\begin{tabular}{|c|c|c|c|c|c|}
\hline & \multirow{2}{*}{ REACIIFS. } & \multirow{2}{*}{\begin{tabular}{|l|} 
TEMPS \\
$\min \times 10$ \\
\end{tabular}} & \multicolumn{2}{|c|}{ VOLUMES $(\mu \mathrm{l})$} & \multirow[t]{2}{*}{ ESSAI } \\
\hline & & & 1 & $\|$ & \\
\hline & $5+$ OF $(x+100)$ & 100 & 160 & 160 & \\
\hline R1 & Mélonge rénctionnel & 75 & 500 & 500 & \\
\hline R2 & Glycerokinose & 65 & 50 & - & \\
\hline 83 & Eou distillee & 34 & - & 50 . & \\
\hline & & & & & \\
\hline & LECTURE & 0 & 710 & 710 & $65 \mathrm{~nm}$ \\
\hline
\end{tabular}

3. Calculs

La concentration en glycérol libre est calculée d’après la formule:

$$
\begin{aligned}
& c(\mathrm{mmol} / \mathrm{l})=\Delta \mathrm{A} \times \frac{1}{\epsilon} \times \frac{\mathrm{VF}}{\mathrm{V}} \\
& \epsilon(\mathrm{NADH}, 365 \mathrm{~nm})=3,4 \times 10^{6} \mathrm{~cm}^{2} \mathrm{~mol}^{-1} \\
& \mathrm{VF}=710 \mu \mathrm{l} \\
& \mathrm{VI}=60 \mu \\
& c=\Delta \mathrm{A} \times 3,48
\end{aligned}
$$

Un coefficient multiplicatif égal à 3,48 est affiché.

\section{Résultats}

\section{Répétabilité et reproductibilité}

La répétabilité est déterminée sur 3 pools de plasma de concentrations différentes en triglycérides. Le coefficient de variation passe de $7 \%$ pour une concentration de $0,35 \mathrm{mmol} / \mathrm{l}$ à moins de $2 \%$ pour une concentration de $2,60 \mathrm{mmol} / \mathrm{l}$ (tab. 3).

Tab. 3. Répétabilité de la méthode enzymatique de dosage des triglycérides adaptée sur GSA II Greiner.

\begin{tabular}{llll}
\hline & Pool 1 & Pool 2 & Pool 3 \\
\hline $\mathrm{n}$ & 24 & 29 & 39 \\
$\mathrm{~m}(\mathrm{mmol} / \mathrm{l})$ & 0,35 & 1,10 & 2,60 \\
$\mathrm{~s}$ & 0,025 & 0,037 & 0,048 \\
$\mathrm{CV}(\%)$ & 7 & 3,33 & 1,86 \\
\hline
\end{tabular}

La reproductibilité est mesurée à l'aide de sérums lyophilisés commerciaux dosés plusieurs fois chaque joụr. Elle ne dépasse paś $6 \%$ (täb. 4).

Tab. 4. Reproductibilité de la méthode de dosage des triglycérides sur GSA II Greiner.

\begin{tabular}{lllll}
\hline & \multicolumn{2}{l}{ Contrôle Hyland } & \multicolumn{2}{l}{ Contrôle Bio-Mérieux } \\
Périóde & $\begin{array}{l}\text { Juillet } \\
1977\end{array}$ & $\begin{array}{l}\text { Septembre } \\
1977\end{array}$ & $\begin{array}{l}\text { Juillet } \\
1977\end{array}$ & $\begin{array}{l}\text { Septembre } \\
1977\end{array}$ \\
\hline n & 183 & 200 & 19 & 21 \\
m (mmol/1) & 1,36 & 1,35 & 1,02 & 0,99 \\
s & 0,073 & 0,054 & $0 ; 059$ & 0,055 \\
CV (\%) & 5,4 & 4 & 5,85 & 5,60 \\
\hline
\end{tabular}

La reproductibilité peut également être évaluée en dosant en double des plasmas de patients. Le coefficient de corrélation entre les résultats est voisin de 1 , ainsi que la pente de la droite de régression.

Le coefficient de variation est de 3,9\% (tab. 5).

Tab. 5. Précision du dosage des trigly cérides sur GSA II Greiner calculée à partir de couples de dosages.

\begin{tabular}{l} 
Nombre de couples \\
Moyenne du premier \\
Moyenne du deuxième \\
Moyenne des différen \\
\hline $\mathbf{t}=-0,2$ (NS) \\
$\mathbf{r}=0,99$ \\
$\mathbf{y}=0,984+0,02$ \\
$\mathrm{CV}=3,9 \%$
\end{tabular}

Dans le cas de la méthode de Soloni, la répétabilité est inférieure à $4,6 \%$ et la reproductibilité à $5,7 \%$. Le coefficient de variation de la méthode de dosage du glycérol libre est inférieur à $3 \%$ dans une série et à $4 \%$ sur une période de trois mois.

\section{Linéarité}

La linéarité a été vérifiée à l'aide de plasmas hyperlipémiques et d'un pool de plasma surchargé en glycérol. Au-delà de $4 \mathrm{mmol} / 1$ de glycérol, il est nécessaire de diluer les échantillons.

\section{Exactitude}

Pour approcher l'exactitude de la méthode enzymatique de dosage des triglycérides, nous avons utilisé plusieurs moyens:

- dosage d'une solution aqueuse de glycérol

- dosage d'une solution aqueuse de trioléine en présence de Triton X-100

- comparaison avec les résultats obtenus par une méthode sélectionnée chimique: la méthode de Soloni (12).

\section{Solutions aqueuses}

Les gammes aqueuses de glycérol donnent des résultats sous-estimés pour des taux inférieurs à $2 \mathrm{mmol} / \mathrm{l}$ et surestimés au-delà. La différence est surtout importante $(-7 \%)$ pour des taux de triglycérides de $0,5 \mathrm{mmol} / 1$ (fig. 1), mais elle reste de l'ordre des variations analytiques. Nous avons retrouvé les valeurs annoncées des solutions aqueuses de trioléine en présence de Triton X-100.

\section{Comparaison avec la méthode chimique de Soloni}

Des sérums témoins du commerce, ainsi que des plasmas de patients ont été dosés à la fois par la méthode enzymatique et la méthode chimique (tab. 6). La valeur du glycérol libre est précisée pour chacun des dosages 


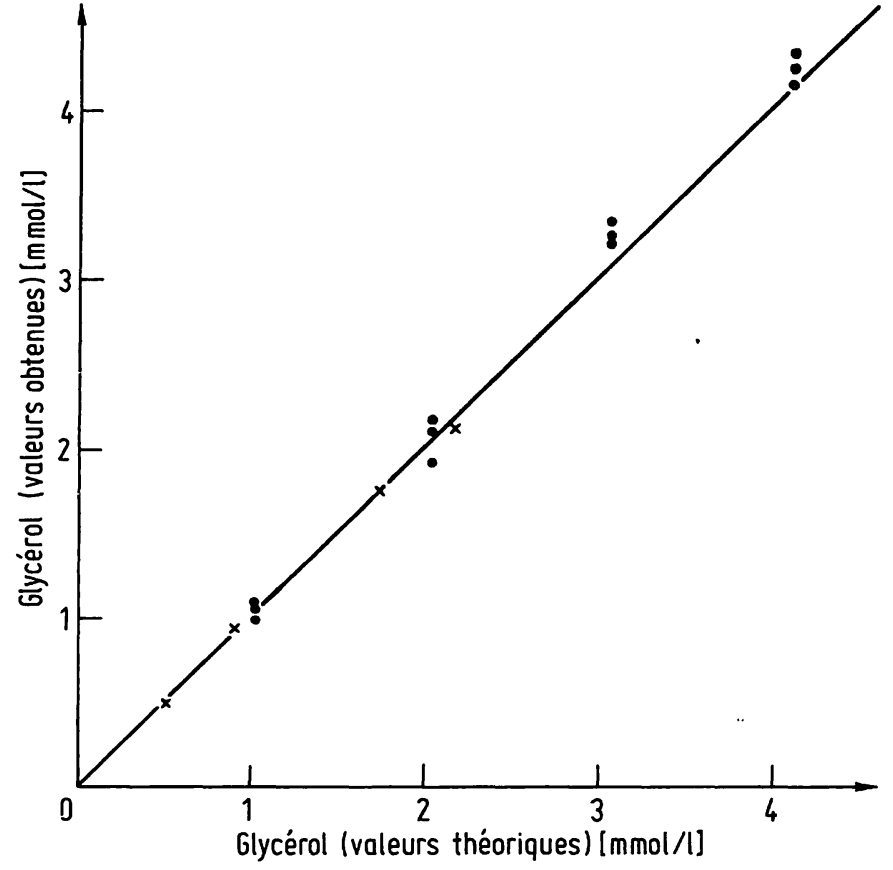

Fig. 1. Exactitude du dosage des triglycérides (mmol/l), gamme aqueuse de gly cérol $(\bullet)$, gamme aqueuse de trioléine $(x)$.

effectués par voie enzymatique. Ce glycérol libre n'interfère pas dans le dosage des triglycérides par la méthode chimique.

Tab. 6. Exactitude de la méthode enzymatique. Comparaison avec la méthode chimique de Soloni (12).

\begin{tabular}{|c|c|c|c|c|}
\hline & \multicolumn{3}{|c|}{ Méthode enzymatique } & \multirow{2}{*}{$\begin{array}{l}\text { Méthode } \\
\text { chimique } \\
\text { tri- } \\
\text { glycérides }\end{array}$} \\
\hline & $\begin{array}{l}\text { glycérol } \\
\text { total }\end{array}$ & $\begin{array}{l}\text { glycérol } \\
\text { libre }\end{array}$ & $\begin{array}{l}\text { tri- } \\
\text { glycérides }\end{array}$ & \\
\hline $\begin{array}{l}\text { Precilip } \\
\text { (mmol/l) }\end{array}$ & $\begin{array}{l}n=18 \\
1,155\end{array}$ & $\begin{array}{l}n=18 \\
0,475\end{array}$ & 0,68 & $\begin{array}{l}n=5 \\
0,64\end{array}$ \\
\hline $\begin{array}{l}\text { Lyotrol } \\
(\mathrm{mmol} / \mathrm{l})\end{array}$ & $\begin{array}{l}n=11 \\
2,26\end{array}$ & $\begin{array}{l}n=11 \\
0,430\end{array}$ & 1,83 & $\begin{array}{l}\mathrm{n}=7 \\
1,80\end{array}$ \\
\hline $\begin{array}{l}\text { Plasmas de } \\
\text { patients adultes } \\
(\mathrm{mmol} / \mathrm{l})\end{array}$ & $\begin{array}{l}n=23 \\
0,78\end{array}$ & $\begin{array}{l}n=23 \\
0,085\end{array}$ & 0,695 & $\begin{array}{l}n=23 \\
0,697\end{array}$ \\
\hline
\end{tabular}

Le glycérol libre est à un taux élevé dans les sérums lyophilisés du commerce $(0,435$ à $0,475 \mathrm{mmol} / \mathrm{l})$. Il peut représenter dans certains cas jusqu'à $70 \%$ de la valeur des triglycérides. Ces concentrations sont plus faibles chez les sujets adultes, en moyenne $0,085 \mathrm{mmol} / 1$. Après déduction de la valeur exacte du glycérol libre, les chiffres des triglycérides mesurés par la méthode enzymatique et par la méthode chimique ne sont pas différents (test de $t$ non significatif, coefficient de corrélation: 0,96 ). Nous avons poursuivi ces essais en dosant le glycérol libre et les triglycérides plasmatiques dans un échantillon d'environ 1200 sujets supposés sains, âgés de 4 à 90 ans.
Les valeurs du glycérol libre observées pour le percentile 50 diminuent avec l'âge de $0,115 \mathrm{mmol} / 1$ entre 4 et 7 ans à $0,055 \mathrm{mmol} / 1$ après 18 ans, alors que pour les mêmes tranches d'âge, les triglycérides augmentent de $0,43 \mathrm{mmol} / 1$ à plus de $0,73 \mathrm{mmol} / 1$. Les proportions de glycérol libre atteignent 12 à $26 \%$ chez les enfants de moins de 16 ans. Au-delà, elles varient de 5 à $10 \%$ suivant le sexe et l'âge (tab. 7).

Tab. 7. Valeurs du glycérol total; du glycérol libre et des triglycérides plasmatiques chez des sujets supposés sains en fonction de l'âge et du sexe (valeurs pour le percentile 50).

\begin{tabular}{lllll}
\hline Age & $\begin{array}{l}\text { glycérol } \\
\text { total }\end{array}$ & $\begin{array}{l}\text { glycérol } \\
\text { libre }\end{array}$ & $\begin{array}{l}\text { tri- } \\
\text { glycé- } \\
\text { rides }\end{array}$ & $\begin{array}{l}\text { Pourcentage } \\
\text { de glycérol } \\
\text { libre par } \\
\text { rapport aux } \\
\text { triglycérides }\end{array}$ \\
\hline Hommes & & & & \\
$4-7$ ans & 0,550 & 0,115 & 0,43 & 26,5 \\
$7-10$ ans & 0,494 & 0,091 & 0,40 & 22,5 \\
$10-13$ ans & 0,543 & 0,078 & 0,46 & 17 \\
$13-16$ ans & 0,554 & 0,064 & 0,49 & 13 \\
$16-18$ ans & 0,629 & 0,050 & 0,58 & 8,5 \\
$18-30$ ans & 0,777 & 0,050 & 0,73 & 7 \\
$30-40$ ans & 0,983 & 0,057 & 0,93 & 6 \\
$40-50$ ans & 1,110 & 0,055 & 1,05 & 5 \\
$50-60$ ans & 1,134 & 0,065 & 1,07 & 6 \\
$>60$ ans & 1,133 & 0,059 & 1,07 & 5,5 \\
& & & & \\
Femmes & & & & \\
$4-7$ ans & 0,546 & 0,108 & 0,44 & 25 \\
$7-10$ ans & 0,558 & 0,096 & 0,46 & 21 \\
$10-13$ ans & 0,661 & 0,084 & 0,58 & 14 \\
$13-16$ ans & 0,641 & 0,070 & 0,57 & 12 \\
$16-18$ ans & 0,578 & 0,069 & 0,51 & 13,5 \\
$18-30$ ans & 0,625 & 0,059 & 0,57 & 10,5 \\
$30-40$ ans & 0,646 & 0,059 & 0,59 & 10 \\
$40-50$ ans & 0,759 & 0,064 & 0,69 & 9 \\
$50-60$ ans & 0,910 & 0,071 & 0,84 & 8,5 \\
$>60$ ans & 1,100 & 0,075 & 1,02 & 7,5 \\
\hline
\end{tabular}

\section{Conservation}

$A-196^{\circ} \mathrm{C}$

Trois pools de plasma frais, à des concentrations différentes en triglycérides, ont été dosés avant congélation et après 1 jour, 2 jours, 9 jours, 13 jours, 1 mois, 3 mois, 6 mois de conservation. La congélation se fait dans les vapeurs d'azote liquide à $-130^{\circ} \mathrm{C}$ et la conservation dans l'azote liquide à $-196^{\circ} \mathrm{C}$. Les résultats correspondant à la moyenne de quatre mesures sont présentés dans le tableau 8.

Le glycérol libre augmente de 6 à $32 \%$ après un jour de conservation, puis les valeurs se stabilisent (tab. 8). L'analyse de la variance permet de comparer la variation analy tique et la variation due à la conservation. Le test F est significatif (au risque 1\%) dans le cas du pool 3 seulement. Pour les pools 1 et 2 , la variation due à la coñservation à $-196^{\circ} \mathrm{C}$ n'est pas significative.

Quant aux triglycérides «vrais», les,variations globales observées s'échelonnent de $-4 \%$ à $+17 \%$ pour le pool 
Tab. 8. Conservation du glycérol libre, du glycérol total et des triglycérides à $-196^{\circ} \mathrm{C}$ (résultats en mmol/1).

\begin{tabular}{|c|c|c|c|c|c|c|c|c|c|}
\hline & $\begin{array}{l}\text { Pool } 1 \\
\text { glycérol } \\
\text { libre }\end{array}$ & $\begin{array}{l}\text { giycérol } \\
\text { total }\end{array}$ & triglycérides & $\begin{array}{l}\text { Pool } 2 \\
\text { glycérol } \\
\text { libre }\end{array}$ & $\begin{array}{l}\text { glycérol } \\
\text { total }\end{array}$ & triglycérides & $\begin{array}{l}\text { Pool } 3 \\
\text { glycérol } \\
\text { libre }\end{array}$ & $\begin{array}{l}\text { glycérol } \\
\text { total }\end{array}$ & $\begin{array}{l}\text { tri- } \\
\text { glycérides }\end{array}$ \\
\hline \multicolumn{10}{|c|}{$\begin{array}{l}\text { Temps de } \\
\text { conservation }\end{array}$} \\
\hline $\begin{array}{c}0 \\
1 \text { jour } \\
2 \text { jours } \\
9 \text { jours } \\
13 \text { jours } \\
1 \text { mois } \\
3 \text { mois } \\
6 \text { mois }\end{array}$ & $\begin{array}{l}0,073 \\
0,096 \\
0,089 \\
0,105 \\
0,102 \\
0,107 \\
0,106\end{array}$ & $\begin{array}{l}0,55 \\
0,615 \\
0,65 \\
0,61 \\
0,57 \\
0,57 \\
0,62\end{array}$ & $\begin{array}{l}0,48 \\
0,52 \\
0,56 \\
0,50 \\
0,47 \\
0,46 \\
0,51\end{array}$ & $\begin{array}{l}0,144 \\
0,152 \\
0,153 \\
0,165 \\
0,156 \\
0,162 \\
0,162 \\
0,155\end{array}$ & $\begin{array}{l}1,18 \\
1,18 \\
1,25 \\
1,21 \\
1,12 \\
1,11 \\
1,26 \\
1,17\end{array}$ & $\begin{array}{l}1,04 \\
1,03 \\
1,10 \\
1,04 \\
0,96 \\
0,95 \\
1,10 \\
1,02\end{array}$ & $\begin{array}{l}0,131 \\
0 ; 173 \\
0,156 \\
0,163 \\
0,155 \\
0,170 \\
0,169 \\
0,155\end{array}$ & $\begin{array}{l}1,74 \\
1,80 \\
1,86 \\
1,66 \\
1,78 \\
1,79 \\
1,87 \\
1,83\end{array}$ & $\begin{array}{l}1,61 \\
1,63 \\
1,70 \\
1,50 \\
1,62 \\
1,62 \\
1,70 \\
1,67\end{array}$ \\
\hline
\end{tabular}

Tab. 9. Conservation du glycérol libre, du glycérol total et des triglycérides à $+4{ }^{\circ} \mathrm{C}$ (résultats en mmol/1).

\begin{tabular}{|c|c|c|c|c|c|c|c|c|c|}
\hline & $\begin{array}{l}\text { Pool } 1 \\
\text { glycérol } \\
\text { libre }\end{array}$ & $\begin{array}{l}\text { glycérol } \\
\text { total }\end{array}$ & triglycérides & $\begin{array}{l}\text { Pool } 2 \\
\text { glycérol } \\
\text { libre }\end{array}$ & $\begin{array}{l}\text { glycérol } \\
\text { totạl }\end{array}$ & triglycérides & $\begin{array}{l}\text { Pool } 3 \\
\text { glycérol } \\
\text { libre }\end{array}$ & $\begin{array}{l}\text { glycérol } \\
\text { total }\end{array}$ & $\begin{array}{l}\text { tri- } \\
\text { glycérides }\end{array}$ \\
\hline $\begin{array}{l}\text { Avant conservation } \\
\text { Après } 4 \text { heures } \\
\text { Après } 1 \text { jour } \\
\text { Après } 2 \text { jours } \\
\text { Après } 5 \text { jours }\end{array}$ & $\begin{array}{l}0,095 \\
0,083 \\
0,085 \\
0,095 \\
0,108\end{array}$ & $\begin{array}{l}0,54 \\
0,53 \\
0,54 \\
0,54 \\
0,59\end{array}$ & $\begin{array}{l}0,45 \\
0,45 \\
0,46 \\
0,45 \\
0,48\end{array}$ & $\begin{array}{l}0,131 \\
0,130 \\
0,142 \\
0,164 \\
0,220\end{array}$ & $\begin{array}{l}1,06 \\
1,04 \\
1,03 \\
1,08 \\
1,20\end{array}$ & $\begin{array}{l}0,93 \\
0,91 \\
0,89 \\
0,92 \\
0,98\end{array}$ & $\begin{array}{l}0,138 \\
0,137 \\
0,166 \\
0,223 \\
0,345\end{array}$ & $\begin{array}{l}1,87 \\
1,84 \\
1,78 \\
1,94 \\
2,10\end{array}$ & $\begin{array}{l}1,73 \\
1,70 \\
1,61 \\
1,72 \\
1,75\end{array}$ \\
\hline
\end{tabular}

1 de concentration $0,48 \mathrm{mmol} / 1$, de $-9 \%$ à $+6 \%$ pour le pool $2(1,04 \mathrm{mmol} / \mathrm{l})$ et de -7 à $+6 \%$ pour le pool $3(1,6 \mathrm{mmol} / \mathrm{l})$ (tab. 8$)$ et ne sont pas significatives.

La stabilité des triglycérides à $-196^{\circ} \mathrm{C}$ est donc satisfaisante. Par contre, le taux de glycérol libre tend à augmenter légèrement au cours de la conservation (de l'ordre de $0,02 \mathrm{mmol} / \mathrm{l}$ ).

\section{$A+4^{\circ} \mathrm{C}$}

Le même protocole que celui utilisé pour l'étude de la conservation des triglycérides à $-196^{\circ} \mathrm{C}$ a été repris pour étudier la conservation à $+4^{\circ} \mathrm{C}$. Trois pools de plasmas de valeurs différentes ont été dosés 5 fois avant conservation, aprè̀s $4 \mathrm{~h}, 1$ jour, 2 jours et 5 jours à $+4^{\circ} \mathrm{C}$. Le glycérol librè varie peu au cours d'une même journée. Le taux augmente de $8 \%$ après 2 jourrs. Il atteint même $150 \%$ après 5 jours de conservation selon la concentration, alors que dans le même temps, le témoin journalier varie de 0,319 à $0,300 \mathrm{mmol} / 1$. L'analyse de la variance confirme que la variation du glycérol libre après 5 joúrs de conservation à $+4^{\circ} \mathrm{C}$ est supérieure à la variation analytique (test de $\mathrm{F}$ significatif pour le risque $1 \%$ ).

Par contre, le taux des triglycérides est plus stable (tab. 9). Le test de F ne devient significatif qư'après 5 jours de conservation des échantillons 1 et 2 .
Interférences analy tiques dues aux médicaments

Nous avons testé l'effet in vitro de différents médicaments en surchargeant un pool de plasma contenant $1,35 \mathrm{mmol} / 1$ de triglycérides avec des solutions de médicaments. Chaque dosage s'accompagne d'un témoin dans lequel seul le médicament a été omis. Les résultats présentés dans le tableau 10 correspondent à la moyenne de trois mesures effectuées sur le plasma surchargé en médicament et le témoin correspondant.

Il n'y a pas de différence significative entre les pools témoins et les pools surchargés. L'acide ascorbique, l'aspirine (acide acétylsalicylique), le clofibrate, le glucophage ( $\mathrm{N}, \mathrm{N}^{\prime}$-dimethylbiguanide [metformin]), l'insoral (phenylethylbiguanide [phenformin]), le procetofène, le phénobarbital n'ont pas d'influence sur le dosage des triglycérides par voie entièrement enzymatique.

\section{Interférence due à l'hém oglobine}

Un pool de plasma frais a été surchargé avec de l'hémoglobine en quantités croissantes. Celle-ci ne provoque pas de variation significative du taux de triglycérides mesurés par méthode enzymatique (tab. 10). 
Tab. 10. Effet in vitro des médicaments et de l'hémoglobine sur le dosage enzymatique des triglycérides.

\begin{tabular}{|c|c|c|c|}
\hline & $\begin{array}{l}\text { Concentration } \\
(\mathrm{mmol} / \mathrm{l})\end{array}$ & $\begin{array}{l}\text { Variation } \\
\text { par rapport } \\
\text { au témoin } \\
(\%)\end{array}$ & $\begin{array}{l}\text { Test de } t \\
(p \\
<0,05)\end{array}$ \\
\hline Acide ascorbique & 0,11 & 1,8 & NS \\
\hline $\begin{array}{l}\text { Acide } \\
\text { acétylsalicylique }\end{array}$ & 1,1 & 1,6 & NS \\
\hline Clofibrate & 12 & 1,8 & NS \\
\hline N.N'dimethylbiguanide & 2,3 & 1,8 & NS \\
\hline Phenylethylbiguanide & 7,3 & 4 & NS \\
\hline Procetoféne & 2,1 & 1 & NS \\
\hline Phénobarbital sodique & 0,8 & 4 & NS \\
\hline Hémoglobine & $\begin{array}{r}26 \\
53 \\
106\end{array}$ & $\begin{array}{l}7 \\
1 \\
7\end{array}$ & $\begin{array}{l}\text { NS } \\
\text { NS } \\
\text { NS }\end{array}$ \\
\hline
\end{tabular}

\section{Comparaison sérum-plasma}

II n'y a pas de différence significative entre les dosages effectués sur sérum et sur plasma (14).

\section{Discussion}

Le GSA II Greiner est un appareil automatique permettant une adaptation très simple de la méthode manuelle de dosage des triglycérides par voie enzymatique. Les réactifs sont stockés à $+4^{\circ} \mathrm{C}$, ce qui favorise leur conservation. La température du bain marie fixée à $37^{\circ} \mathrm{C}$ est stable (CV: $0,3 \%$ ). Nous avons sensibilisé la méthode proposée par le constructeur en utilisant un volume de liquide biologique plus important: $20 \mu l$ au lieu de $10 \mu l$.

La répétabilité est inférieure à $4 \%$ dans la zone des valeurs moyennes de la triglycéridémie, inférieure à $2 \%$ dans la zone des valeurs élevées. Elle est de $7 \%$ dans la zone des valeurs faibles $(0,35 \mathrm{mmol} / \mathrm{l})$. La reproductibilité est de $5,8 \%$ pour une triglycéridémie physiologique. Cette précision est tout à fait acceptable pour le dosage des triglycérides. Elle est du même ordre qu'avec d'autres appareils automatiques $(5,6,8,9,10$, $15,16)$. En manuel, par contre, le coefficient de variation dépend beaucoup du manipulateur et peut atteindre $11,6 \%(17)$. Il faut rappeler toutefois que les chiffres cités englobent à la fois la précision due à l'appareillage et celle due aux réactifs.

L'exactitude de la méthode enzymatique a été étudiée par comparaison avec la méthode de Soloni (12) qui, par son principe même, est spécifique des triglycérides (18). En effet, plus de $99 \%$ des triglycérides sont extraits par le nonane et seulement $1,3 \%$ des phospho- lipides. Le glucose et le glycérol n'interfèrent pas (18). Après dosage et déduction du glycérol libre, les résultats de la méthode enzymatique concordent parfaitement avec la méthode de Soloni. Le coefficient de corrélation est de 0,96 et les différences non significatives.

Le taux de glycérol libre présent dans les sérums lyophilisés est supérieur à $0,4 \mathrm{mmol} / 1$. Cette valeur explique les différences obtenues au cours des contrôles de qualité interlaboratoires entre les méthodes chimiques et enzymatiques, si le glycérol libre n'est pas déduit des résultats de cette dernière $(17,19)$.

De plus, ainsi que l'avaient déjà suggéré Revol et al (21), la mesure du glycérol libre, présent dans le plasma des patients, devrait être effectué systématiquement, dans un souci d'exactitude du résultat final du dosage des triglycérides. La déduction de $0,1 \mathrm{mmol} / 1$, préconisée par certains auteurs (22) ne se justifie pas cheż les sujets diabétiques, alcooliques (19), hyperthyroïdiens (20) ou chez les enfants.

Nous avons vérifié que les médicaments hypolipémiants les plus couramment prescrits (procétofène, clofibrate), certains hypoglycémiants (glucophage, insoral), le phénobarbital, l'aspirine, l'acide ascorbique ne modifient pas les résultats de la méthoḍe enzymatique. Muller et al (23) ont étudié l'influence de nombreux médicaments: ils n'ont trouvé aucune interférence des antigoutteux (allopurinol, probénécide), des tranquillisants (chlorodiazepoxide, doxepine), des anti-inflammatoires (indométacine, acide nifluminique, oxyphénbutazone). Nous avons montré également que l'hémoglobine à un taux de $1,7 \mathrm{~g} / 1, l^{\prime}$ héparinate de lithium utilisé comme anticoagulant, n'interfèrent pas dans le dosage. Wakayama et al (24) ont montré, par ailleurs, que les phospholipides et l'acide lactique n'avaient aucune influence sưr le résultat final.

La stabilité de la conservation des triglycérides en fonction du temps et de la température a fait l'objet de nombreuses publications parfois contradictoires. Les triglycérides dosés par méthode chimique (25) sont stables 3 jours à température ambiante ou à $4{ }^{\circ} \mathrm{C}(26)$. Par contre, le glycérol libre peut augmenter jusqu'à $64 \%$ après $24 \mathrm{~h}$ à $25^{\circ} \mathrm{C}(27)$. A $4{ }^{\circ} \mathrm{C}$, il est stable 3 jours (22). Nous avons montré qu'à $4^{\circ} \mathrm{C}$, la concentration en glycérol libre est stable 1 jour et augmente de $8 \%$ après 2 jours et jusqu'à une foie et demie la vāleur initiale, après 5 jours. La quantité de glycérol total augmente parallèlement, de telle sorte que le taux des triglycérides reste stable. Il faut donc conclure à une libération progressive de glycérol à partir de composés autres que les triglycérides, en particulier des phospholipides (11).

L'exemple de la conservation des triglycérides vient à nouveau appuyer la remarque faite précédemment de doser le glycérol libre dans les plasmas ayant séjourné plus de 2 jours à $+4^{\circ} \mathrm{C}$. En effet; la mesure du glycérol total est entachée d'une erreur par excès. 
La congélation des plasmas à $-196^{\circ} \mathrm{C}$, dans l'azote liquide, permet une excellente conservation à long terme du glycérol libre et des triglycérides.

\section{Conclusion}

Les performances du GSA II Greiner pour le dosage des triglycérides par voie entièrement enzymatique sont tout à fait satisfaisantes: répétabilité de l'ordre de $3 \%$ et reproductibilité de $4 \%$ dans la zone des valeurs fréquentes de $1 \mathrm{mmol} / \mathrm{l}$. La linéarité est limitée à une

\section{Bibliographie}

1. Bucolo, G. \& David, H. (1973), Clin. Chem. 19, 476-482.

2. Coudon, B., Bouige, D. \& Giraudet, P. (1974), Clin. Chim. Acta 55, 129-138.

3. Bucolo, G., Yabut, J. \& Chang, T. Y. (1975), Clin. Chem. $21,420-424$.

4. Vlastelica, D., Bucolo, G., Yabut, J. \& Kessle, R. G. (1974), Clin. Chem. 20, 907.

5. Dingeon, B., Roullet, M. J. \& Roullet, A. (1974), Ann. Biol. Clin. 32, 127-134.

6. Chong-Kit, R. \& McLaughlin, P. (1974), Clin. Chem. 20, 1454-1457.

7. Olson, A. D. \& Brennan, R. L. (1973), Clin. Chem. 19, 1060-1062.

8. Wakayama, J. E. \& Swanson, J. R. (1974), Clin. Chem. 20, 868-871.

9. Ziegenhorn, J. (1975), Clin. Chem. 21, 1627-1629.

10. Dicesare, J. L. (1975), Clin. Chem. 21, 1448-1451.

11. Tiffany, T. O., Morton, J. M., Hall, E. M. \& Garett, A. S. (1974), Clin. Chem. 20, 476-481.

12. Soloni, F. (1971), Clin. Chem. 17, 529-534.

13. Kreutz, F. H. (1962), Klin. Wochenschr. 40, 362-363.

14. Henny, J., Houot, O., Steinmetz, J. \& Siest, G. (1976), Ann. Biol. Clin. 34, 335-340.

15. Großmann, S. H., Mollo, E. \& Ertingshausen, G. (1976), Clin. Chem. 22, 1310-1313. concentration de $4 \mathrm{mmol} / 1$. L'exactitude est comparable à celle obtenue avec la méthode chimique de Soloni (12). Une attention particulière doit être apportée toutefois à la déduction du taux réel du glycérol libre et non pas à celle d'un chiffre arbitraire. Cela suppose son dosage systématique en parallèle avec celui des triglycérides. Lorsque ces précautions sont respectées, nous avons pu démontrer la bonne stabilité des triglycérides pendant une conservation de 5 jours à $+4^{\circ} \mathrm{C}$ et de 6 mois dans l'azote liquide, ainsi que la spécificité du dosage.

16. Rietz, E. B. \& Guilbault, G. G. (1977), Clin. Chem. 23, 286-288.

17. Carter, T., Martin, P. J., Wilding, P. \& Goldberg, D. M. (1976), Clin. Chem. 22, 520-524.

18. Giegel, J., Soloni, F., Trinidad, E., Cohen, B. \& Clema, W. (1975), Clin. Chem. 21, 1575-1581.

19. Carter, T. \& Wilding, P. (1976), Clin. Chim. Acta 70 , 433-447.

20. Jonesowen, V. M. (1976), Clin. Biochem. 9, 99-103.

21. Revol, A., Trouyez, R. \& Piccardi, G. (1972), in Contrôle de qualité des examens de laboratoire, Lyon, 149-156.

22. Stinshoff, K., Weisshaar, D., Staehler, F., Hesse, D., Gruber, W. \& Steier, E. (1977), Clin. Chem. 23, 1029-1032.

23. Müller, P. H., Schmülling, R. M., Liebich, H. M., Eggstein, M., Stahler, F. \& Stinshoff, K. (1977), J. Clin. Chem. Clin. Biochem. 15, 457-464.

24. Wakayama, J. E. \& Swanson, J. R. (1977), Clin. Chem. 23, 223-228.

25. Neri, B. \& Frings, C. (1973), Clin. Chem. 19, 1201-1202.

26. Frings, C. S., Neri, B. P., Freeman, K. \& Fendley, T. W. (1974), Clin. Chem. 20, 87-88.

27. Rehkamper, H. (1974), Clin. Chem. 20, 88.

\section{J. Steinmetz}

Laboratoire du

Centre de Médecine Préventive

2, avenue du Doyen Jacques Parisot

F-54500 Vandoeuvre-les-Nancy, France 
,

$$
\text { . }
$$

